\title{
Síndrome hemolítico-urêmica relacionada à infecção invasiva pelo Streptococcus pneumoniae
}

\author{
Hemolytic-uremic syndrome complicating invasive pneumococcal disease
}

Anna Leticia de O. Cestari', Ricardo Vilela², Juliana Kunisawa', Carlos Eduardo Lopes ${ }^{3}$

\section{RESUMO}

Objetivo: A doença pneumocócica é importante problema de saúde pública e raramente há associação desta infecção com a síndrome hemolítico-urêmica (SHU) grave. O objetivo deste artigo é relatar o caso de um paciente com esta associação.

Descrição do caso: Criança do sexo masculino, com 17 meses de idade, admitida no hospital com insuficiência respiratória aguda e necessitando de suporte ventilatório. $\mathrm{O}$ exame radiológico mostrava extensa opacidade homogênea em hemitórax direito. A hemocultura foi positiva para Streptococcus pneumoniae. Nos exames de admissão, notaram-se: hemoglobina de $6,5 \mathrm{~g} / \mathrm{dL}, 38.000$ plaquetas $/ \mathrm{mm}^{3}$, uréia de $79 \mathrm{mg} / \mathrm{dL}$ e creatinina de $1,64 \mathrm{mg} / \mathrm{dL}$. No primeiro dia, apresentou oligoanúria e hipervolemia, necessitando de hemodiafiltração. Evoluiu com disfunção de múltiplos órgãos e óbito no sétimo dia. A necrópsia mostrou áreas extensas de necrose cortical e tubular renal, com depósito de fibrina nas arteríolas.

Comentários: A SHU associada ao pneumococo apresenta morbidade e mortalidade elevadas. Em crianças com doença pneumocócica invasiva e acometimento hematológico ou renal grave, deve-se estar atento a esta rara complicação. Merecem investigação os seguintes aspectos relacionados à doença: a função da detecção precoce de antígenos T ativados no diagnóstico e terapêutica, o papel do fator $\mathrm{H}$ na patogênese, o método ideal de substituição renal e a definição do prognóstico em longo prazo.

Palavras-chave: síndrome hemolítico-urêmica; Streptococcus pneumoniae; neuraminidase.

\section{ABSTRACT}

Objective: Pneumococcal diseases are a major public health problem. Severe hemolytic-uremic syndrome is an uncommon complication. The aim of this study is to report a child with this complication.

Case description: A male child with 17 months old was admitted to the hospital, due to acute respiratory failure, needing ventilatory support. Roentgenogram demonstrated massive condensation of right lung and Streptococcus pneumonia was isolated from blood cultures. Laboratory tests showed hemoglobin level of $6.5 \mathrm{~g} / \mathrm{dL}$, 38,000 platelets $/ \mathrm{mm}^{3}$, blood urea nitrogen of $79 \mathrm{mg} / \mathrm{dL}$ and creatinine of $1.64 \mathrm{mg} / \mathrm{dL}$. On the first day, patient developed oliguria and hypervolemia and needed hemodiafiltration. Multiple organs dysfunction syndrome was followed by death on the seventh day. Necropsy showed extensive renal cortical and tubular necrosis with fibrin deposits on arterioles.

Comments: Hemolytic-uremic syndrome complicating invasive pneumococcal disease has high morbidity and mortality rates. Children with pneumococcal infection and severe hematologic or renal abnormalities should be investigated. The usefulness of early recognition of T-antigen activation on diagnosis and therapeutics, the role of complement factor $\mathrm{H}$ in the pathology, the ideal renal replacement method and the definition of long term outcome are issues to be investigated.

Key-words: hemolytic-uremic syndrome; Streptococcus pneumoniae; neuraminidase.
${ }^{1}$ Pediatra e médica residente da Unidade de Terapia Intensiva Pediátrica (UTIP) do Hospital de Clínicas (HC) da Faculdade de Ciências Médicas (FCM) da Universidade Estadual de Campinas (Unicamp), Campinas, SP, Brasil ${ }^{2}$ Pediatra, médico assistente da UTIP do HC/FCM-Unicamp e mestre em Saúde da Criança e do Adolescente pela FCM-Unicamp, Campinas, SP, Brasil ${ }^{3}$ Pediatra e coordenador da UTIP do HC/FCM-Unicamp, Campinas, SP, Brasil
Endereço para correspondência:

Anna Leticia de O. Cestari

Rua Santos Dumont, 224, apto. 1.003

CEP 13024-020 - Campinas/SP

E-mail: annaletscestari@hotmail.com

Recebido em: 25/10/2007

Aprovado em: 15/1/2008 


\section{Introdução}

A síndrome hemolítico-urêmica (SHU) é uma das principais causas de insuficiência renal aguda em crianças. Segundo o Centers for Disease Control and Prevention, a SHU pode ser definida pela presença de trombocitopenia (contagem de plaquetas inferior a $150.000 / \mathrm{mm}^{3}$ ), início agudo de anemia com sinais de hemólise microangiopática em esfregaço de sangue periférico e por lesão renal aguda evidenciada por hematúria, proteinúria ou aumento do nível sérico de creatinina ${ }^{(1)}$.

A incidência de SHU varia entre 0,2 e 3,4/100.000 a cada ano. Cerca de $90 \%$ dos casos de SHU são precedidos por um quadro de gastrenterite aguda, sendo mais de $80 \%$ após infecção por Escherichia coli, produtora de toxina do tipo Shiga (E. coli O157:H7). Um grupo menor, chamado de SHU atípica, pode se relacionar ao uso de fármacos, herança genética autossômica recessiva ou dominante, gravidez, transplante de órgãos e infecções. A infecção invasiva por Streptococcus pneumoniae (S. pneumoniae) é uma das causas associadas à gênese da SHU neste grupo de pacientes ${ }^{(2)}$.

A síntese de neuraminidase pelo $S$. pneumoniae parece ter papel fundamental na fisiopatologia da SHU relacionada ao S. pneumoniae (SHU-SP). A neuraminidase é uma enzima produzida pela bactéria que quebra o ácido n-acetil-neuramínico, constituinte das glicoproteínas das membranas celulares das células endoteliais, plaquetas, hemácias e células glomerulares. Com a remoção do ácido n-acetil-neuramínico da superfície celular, há exposição do antígeno de Huebner-Thomsen-Friedenreich (ou antígeno T), presente na superfície celular e normalmente encoberto pelo ácido n-acetil-neuramínico ${ }^{(3-5)}$. A maioria dos indivíduos possui anticorpos naturais IgM anti-T circulantes. Originalmente, acreditava-se que a ligação destes anticorpos ao antígeno $\mathrm{T}$ promovesse lesão endotelial, hemólise e plaquetopenia, explicando a patogênese da doença. Todavia, os anti-T são anticorpos frios e incapazes de causar aglutinação in vivo. Assim, a verdadeira patogênese é desconhecida.

Os fatores $\mathrm{H}$ e I e a proteína cofator de membrana (MCP) são reguladores da atividade do complemento e sua perda de função implica maior ativação do complemento. A desregulação do complemento por mutação nos genes que codificam esses fatores está envolvida na patogênese da SHU atípica familiar $^{(6)}$. É interessante notar que a neuraminidase tem capacidade de bloquear o efeito inibitório do complemento pelo fator $\mathrm{H}$ em glóbulos vermelhos humanos, tornando-os vulneráveis à lise induzida pelo complemento ${ }^{(7)}$.

A doença pneumocócica, por sua vez, é um importante problema de saúde pública em todo o mundo ${ }^{(8)}$. A SHU é uma complicação rara da doença pneumocócica invasiva. $\mathrm{A}$ incidência em alguns estudos é inferior a $0,4 \%{ }^{(3)}$. Uma extensa revisão da literatura encontrou menos de 50 casos de SHU-SP já relatados ${ }^{(4)}$. Apesar de rara, a SHU-SP apresenta alta morbidade e mortalidade. Devido à raridade e à gravidade do quadro os autores relatam um caso de SHU relacionada ao $S$. pneumoniae.

\section{Descrição do caso}

Paciente do sexo masculino, 17 meses de idade, branco, previamente hígido, foi admitido em hospital universitário com febre e feridas na boca há sete dias, além de tosse e dificuldade respiratória há três dias. Um dia antes da internação, apresentou piora do estado geral, inapetência, desconforto respiratório e anúria durante 14 horas. Ao exame físico inicial, a criança mostrava-se descorada, com palidez cutânea, mucosas secas, taquicardia, taquipnéia, pressão arterial normal, murmúrio vesicular diminuído em hemitórax direito, com batimento de aletas nasais, uso da musculatura acessória e gemência. Os exames laboratoriais de entrada encontram-se na Tabela 1 . O exame radiológico de

Tabela 1 - Evolução dos exames laboratoriais de criança com SHU

\begin{tabular}{lcccccccc}
\hline Dias de internação & $\mathbf{1}^{\mathbf{0}}$ & $\mathbf{2}^{\circ}$ & $\mathbf{3}^{\circ}$ & $\mathbf{4}^{\circ}$ & $\mathbf{5}^{\circ}$ & $\mathbf{6}^{\circ}$ & $\mathbf{7}^{\circ} \mathbf{a}$ & $\mathbf{7}^{\circ} \mathbf{b}$ \\
\hline Hemoglobina $(\mathrm{g} / \mathrm{dL})$ & 6,5 & 9,1 & 7,7 & 9,7 & 8,6 & 7,8 & 10,9 & 7,3 \\
Hematócrito $(\%)$ & 19,9 & 26,3 & 22,2 & 28,4 & 26,2 & 22,8 & 34,4 & 24,6 \\
Leucócitos $/ \mathrm{mm}^{3}$ & 6.400 & 11.060 & 10.073 & 12.180 & 13.220 & 13.000 & 12.370 & 38.150 \\
Plaquetas $/ \mathrm{mm}^{3}$ & 38.000 & 15.000 & 27.000 & 57.000 & 55.000 & 112.000 & 112.000 & 132.000 \\
Reticulócitos (\%) & - & - & - & - & - & - & - & 0,7 \\
$\mathrm{R}$ & 3,64 & 1,24 & - & 1,33 & 1,41 & 2,18 & 2,21 & - \\
$\mathrm{RNI}$ & 1,58 & 1,33 & - & 1,59 & 2,0 & 3,88 & 4,99 & - \\
Creatinina $(\mathrm{mg} / \mathrm{dL})$ & 1,64 & 3,26 & 3,92 & - & 5,58 & 6,42 & - & 2,41 \\
Uréia $(\mathrm{mg} / \mathrm{dL})$ & 79 & 135 & 139 & - & 166 & 192 & - & 76 \\
\hline
\end{tabular}

$\mathrm{R}=$ razão para tempo de protrombina; $\mathrm{RNI}=$ relação normatizada internacional para tempo de tromboplastina 
tórax mostrou extensa opacidade homogênea em hemitórax direito sem sinais de derrame pleural. Devido ao quadro de insuficiência respiratória, optou-se por intubação orotraqueal e ventilação mecânica e foi iniciada a antibioticoterapia com ampicilina intravenosa. A hemocultura colhida à admissão mostrou crescimento de $S$. pneumoniae sensível à penicilina. O líquor apresentava-se sem anormalidades.

Durante o primeiro dia de internação, a criança evoluiu com oligúria, mesmo após receber solução expansora e dobutamina. Observou-se também taquicardia, pressão arterial e venosa central ascendentes, anasarca, aumento da hepatimetria e piora respiratória, com necessidade de pressões elevadas à assistência ventilatória. Recebeu furosemida intravenosa intermitente e contínua sem resposta, evoluindo para anúria nas primeiras 24 horas de internação. Foram repetidos os exames laboratoriais, os quais evidenciaram plaquetopenia, anemia, hemácias crenadas, células em alvo, esquisócitos e esferócitos (Tabela 1). Recebeu concentrado de hemácias três vezes durante a internação. $\mathrm{O}$ paciente foi submetido à diálise peritoneal, que não resultou em melhora da hipervolemia nem impediu o aumento das escórias nitrogenadas, sendo substituída por hemodiafiltração contínua.

No quinto dia de internação, evoluiu com piora progressiva do quadro respiratório, apresentando pneumatocele volumosa em hemitórax direito, com necessidade de drenagem torácica. Houve aumento progressivo das escórias nitrogenadas e coagulopatia de consumo (Tabela 1). No sexto dia de internação, mostrava instabilidade hemodinâmica, com queda da pressão arterial e necessidade de vasopressor, além de evolução para hiperglicemia refratária, acidose metabólica grave, hipercloremia e deterioração

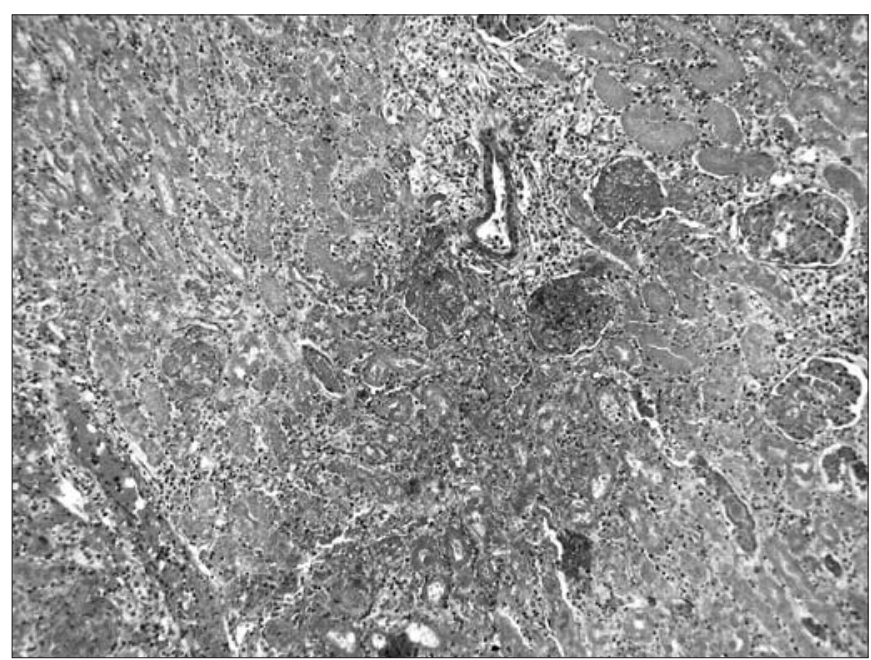

Figura 1 - Análise histológica de fragmento renal com extensa necrose cortical e tubular neurológica, culminando com insuficiência de múltiplos órgãos e óbito no sétimo dia.

A necropsia mostrou áreas de necrose em toda superfície renal bilateralmente e, à microscopia, áreas extensas de necrose cortical e tubular com depósito de fibrina nas arteríolas (Figuras 1 e 2).

\section{Comentários}

O diagnóstico da SHU-SP requer evidência microbiológica de doença pneumocócica invasiva, ou seja, isolamento do microrganismo em cultura de sangue ou de outras secreções corpóreas (líquor, líquido pleural, peritoneal, pericárdico e articular), exceto aspirados traqueais, de seios da face e de orelha média. A presença de infecção pneumocócica invasiva e de microangiopatia trombótica em biópsia renal ou necropsia são considerados como evidências definitivas da SHU ${ }^{(3,9)}$. No caso relatado, a hemocultura colhida à admissão apresentou crescimento de $S$. pneumoniae.

A associação da SHU relacionada ao $S$. pneumoniae com pneumonia é mais freqüente do que com meningite, correspondendo a 65 e $28 \%$ dos casos relatados, respectivamente. A associação da SHU relacionada à bacteremia por $S$. pneumoniae responde por somente $7 \%$ dos pacientes relatados $^{(4)}$. A complicação extra-renal mais encontrada em pacientes com SHU-SP é o empiema ${ }^{(3,4)}$. Este achado sugere que a doença supurativa pode aumentar o risco para SHU-SP, ao menos nos casos associados à pneumonia.

Do ponto de vista histopatológico, a SHU pode ser classificada em três grupos, de acordo com o tipo de lesão renal: necrose cortical, predominância de lesão glomerular ou pre-

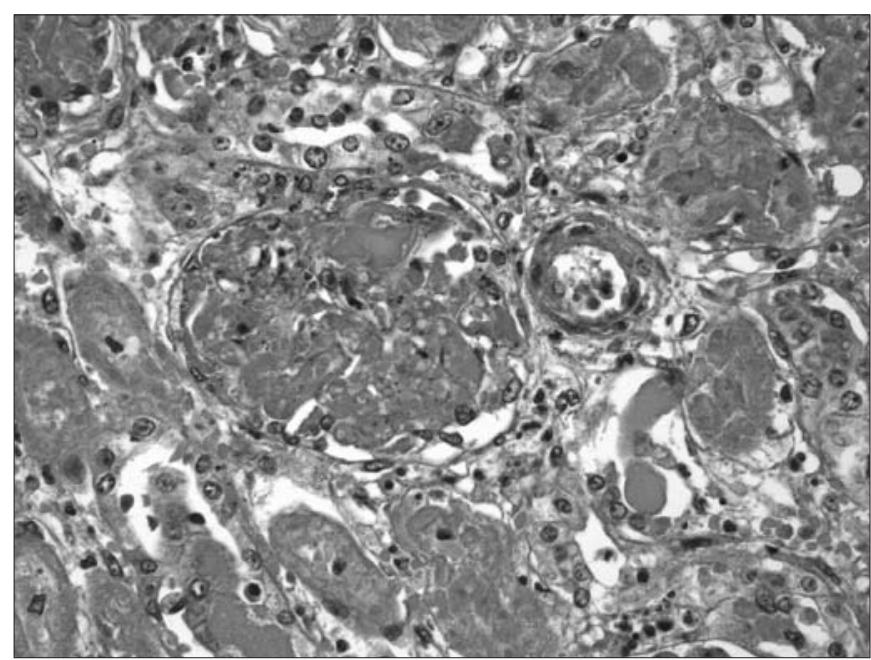

Figura 2 - Análise histológica da lesão renal com depósito de fibrina em arteríola 
dominância de lesão arteriolar ${ }^{(10)}$. É notada concomitância de lesão glomerular e arteriolar. A lesão mais significativa nas formas atípicas é a arteriolar. No caso descrito, a necropsia mostrou áreas de necrose cortical e lesão arteriolar.

$\mathrm{O}$ intervalo entre o início dos sintomas da doença pneumocócica e o desenvolvimento da SHU varia de um dia a duas semanas - uma semana, em média ${ }^{(3)}$. No caso descrito, o diagnóstico de pneumonia pneumocócica foi realizado de forma simultânea ao aparecimento da SHU-SP.

A maioria dos casos de SHU-SP é referida em crianças menores de dois anos. A incidência de doença pneumocócica invasiva é maior nesta faixa etária. Além disso, a ativação do antígeno T ocorre mais freqüentemente em criança menores, o que sugere que variações da reação antígeno-anticorpo relacionadas à idade podem contribuir para essa tendência ${ }^{(11)}$. Todos os $S$. pneumoniae produzem neuraminidase ${ }^{(5,12,13)}$. No entanto, nem toda infecção pneumocócica resulta em ativação do antígeno $\mathrm{T}^{(13)}$. Diferentes sorotipos de $S$. pneumoniae podem produzir neuraminidases com diversos graus de atividades e concentrações, expondo quantidades variáveis de antígeno T ativado. Fatores do hospedeiro, como a quantidade de antígeno T, podem também influenciar no tipo de resposta ${ }^{(3)}$. Alguns indivíduos apresentam bactérias na flora intestinal que expressam antígenos semelhantes ao antígeno $\mathrm{T}$, o que estimula a síntese de anticorpos naturais anti- $\mathrm{T}^{(13,14)}$.

A identificação precoce do antígeno $T$ ativado nos pacientes com doença pneumocócica invasiva parece ser relevante para a prática clínica. Um estudo que reuniu 36 crianças com doença pneumocócica invasiva detectou a ativação do antígeno $\mathrm{T}$ em $69 \%$ dos casos. Destes, 52\% desenvolveram SHU e 24\%, anemia hemolítica. No grupo em que não foi detectado o antígeno T, nenhum caso de SHU foi identificado e, em $27 \%$ deles, foi observada anemia hemolítica. Estas diferenças foram estatisticamente significantes ${ }^{(14)}$. Neste estudo, os autores também demonstraram que a ativação do antígeno $\mathrm{T}$ ocorre previamente à queda de hemoglobina e plaquetas. Assim, a detecção do antígeno $T$ ativado pode ser um indicador precoce da gravidade da doença pneumocócica invasiva ${ }^{(14)}$.

As investigações mais recentes associam a SHU atípica à inativação de fatores inibidores do complemento(6). É possível que a neuraminidase promova esta inativação na SHU-SP ${ }^{(7)}$. Assim, é possível que a detecção do antígeno $T$ ativado possa vir a ser usada como um marcador indireto da atividade da neuraminidase.
Não há diferença significativa no quadro clínico entre os pacientes que receberam ou não antibiótico antes do diagnóstico de $\mathrm{SHU}^{(4)}$. Da mesma forma, a resistência do S. pneumoniae a antibióticos beta-lactâmicos parece não alterar o curso da doença ${ }^{(5,14)}$.

O tratamento baseia-se no manuseio da insuficiência renal aguda e dos distúrbios hematológicos. Não há tratamento específico para SHU ${ }^{(15)}$. Em 75 a $100 \%$ dos casos de SHU, há indicação de diálise para manejo da insuficiência renal aguda. O tempo médio de diálise varia de dez a 32 dias $^{(3,5,16)}$. Nenhum estudo tem comparado a eficácia dos vários métodos dialíticos na SHU. A escolha do método dialítico na SHU deve ser realizada considerando as vantagens e desvantagens de cada um e o quadro clínico da criança. Em pacientes com SHU, a diálise peritoneal pode ser uma boa escolha e sua instituição precoce é capaz de diminuir a morbimortalidade da doença $a^{(15,17)}$.

Alguns autores recomendam o uso hemácias e plaquetas lavadas e o emprego cauteloso de plasma fresco congelado, evitando-se a oferta de anticorpos naturais anti-T, o que parece estar relacionado à exacerbação dos mecanismos de lesão endotelial ${ }^{(3-5,11,13)}$. A identificação precoce do antígeno T ativado nos pacientes com doença pneumocócica poderia auxiliar na administração de hemoterápicos, recomendando-se o uso de hemácias e plaquetas lavadas ${ }^{(13)}$. No entanto, considerando-se o possível papel do efeito inibidor da neuraminidase sobre o fator $\mathrm{H}$ na patogênese da SHU-SP, esta conduta agora é questionada ${ }^{(7)}$.

A mortalidade na SHU-SP varia de 29 a $50 \%{ }^{(13)}$. De 8 a $30 \%$ dos casos relatados de SHU-SP evoluíram para insuficiência renal crônica entre quatro e 17 anos após o quadro agudo $^{(3)}$. Foi realizado transplante renal em três casos descritos de SHU-SP. No entanto, a seqüela renal após a SHU-SP ainda é um assunto controverso na literatura ${ }^{(3)}$.

Em crianças com doença pneumocócica invasiva e acometimento hematológico ou renal graves, é preciso estar atento ao diagnóstico de SHU. O reconhecimento do quadro e a instituição precoce de diálise podem aumentar a taxa de sobrevivência destes pacientes.

\section{Agradecimentos}

Os autores agradecem ao doutor Marcos Antonio de Paolis pelo apoio incondicional à publicação deste artigo. 


\section{Referências bibliográficas}

1. Centers for Disease Control [homepage on the Internet]. Hemolytic uremic syndrome, post-diarrheal (HUS): 1996, case definition [cited 2007 Feb 04] Available from: http://www.cdc.gov/epo/dphsi/casedef/hemolyticcurrent.htm.

2. Furusawa EA, Koch VH, Kim CA, Fujimura M, Saldanha LB, Okay Y. Síndrome hemolítico-urêmica atípica na criança. J Bras Nefrol 1997;19:280-4.

3. Huang $\mathrm{YH}$, Lin TY, Wong KS, Huang YC, Chiu $\mathrm{CH}$, Lai SH et al. Hemolytic uremic syndrome associated with pneumococcal pneumonia in Taiwan. Eur J Pediatr 2006;165:332-5.

4. Brandt J, Wong C, Mihm S, Roberts J, Smith J, Brewer E et al. Invasive pneumococcal disease and hemolytic uremic syndrome. Pediatrics 2002;110:371-6

5. Cabrera GR, Fortenberry JD, Warshaw BL, Chambliss CR, Butler JC, Cooperstone BG. Hemolytic uremic syndrome associated with invasive Streptococcus pneumoniae infection. Pediatrics 1998;101:699-703.

6. Atkinson JP, Goodship TH. Complement factor $\mathrm{H}$ and the hemolytic uremic syndrome. J Exp Med 2007;204:1245-8.

7. Loirat C, Taylor CM. Hemolytic uremic syndromes. In: Avner ED, Harmon WE, Niaudet $P$, editors. Pediatric nephrology. $5^{\text {th }}$ ed. Philadephia: Lippincott Williams and Wilkins; 2004. p. 887-915.

8. Jernigan DB, Cetron MS, Breiman RF. Minimizing the impact of drug-resistant Streptococcus pneumoniae (DRSP). A strategy from the DRSP Working Groups. JAMA 1996;275:206-9.
9. Proulx F, Sockett P. Prospective surveillance of Canadian children with the haemolytic uraemic syndrome. Pediatr Nephrol 2005;20:786-90.

10. Goldraich NP. Síndrome hemolítico-urêmica e suas diferentes formas de apresentação: uma patologia atual da Nefrologia. J Bras Nefrol 1997;19:294-300.

11. Lee CF, Liu SC, Lue KH, Chen JP, Sheu JN. Pneumococcal pneumonia with empyema and hemolytic uremic syndrome in children: report of three cases. J Microbiol Immunol Infect 2006;39:348-52.

12. Barit G, Sakarcan A. Antibiotic resistant Streptococcus pneumoniae and hemolytic uremic syndrome. Eur J Pediatr 2005;164:414-6.

13. Cochran JB, Panzarino VM, Maes LY, Tecklenburg FW. Pneumococcus-induced T-antigen activation in hemolytic uremia syndrome and anemia. Pediatr Nephrol 2004;19:317-21.

14. Huang DT, Chi H, Lee HC, Chiu NC, Huang FY. T-antigen activation for prediction pneumococcus-induced hemolytic uremic syndrome and hemolytic anemia. Pediatr Infect Dis J 2006;25:608-10.

15. Amirlak I, Amirlak B. Haemolytic uraemic syndrome: an overview. Nephrology (Carlton) 2006;11:213-8

16. Nathanson S, Deschenes G. Prognosis of Streptococcus pneumoniae-induced hemolytic uremic syndrome. Pediatr Nephrol 2001;16:362-5.

17. Strazdins V, Watson AR, Harvey B; European Pediatric Peritoneal Sialysis Working Group. Renal replacement therapy for acute renal failure in children: European guidelines. Pediatr Nephrol 2004;19:199-207. 